\title{
Ahkärz̧ungen and ettteratur.
}

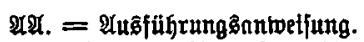

$\mathfrak{2}$ :

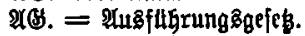

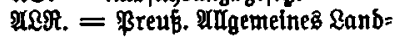
rewt.

$\mathfrak{A P R}$. = anberer Detnung.

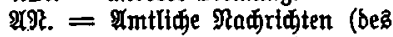

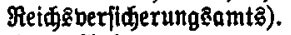

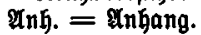

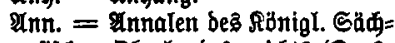
fif ben) $98.1-18$.

aD. = Irbeits oronung.

Appelius = Betwerbeorboung bon 5. Appelius, 1893.

$\mathfrak{A r t .}=\mathfrak{A r t i f e l}$.

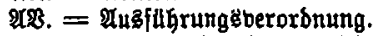

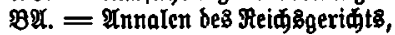
heraugagegef. von Blum u. Braun.

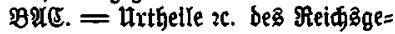
in Eivilladen, von Dr. Blum.

$\mathfrak{B a s .}=\mathfrak{B a b i f}$.

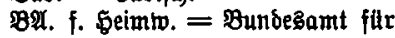
Setmatmejen.

Bayr. $=$ Pagrild.

Bayr. Bษg. 1 ff. $=$ offizielle Eammlung bon Entidetbungen

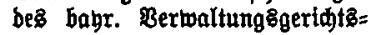
hof

Begr. = Begrïnoung, eineả Be= ieşę.

Bet. = Belanntmadung.

Bem. $=$ Bemering.

Ber. = Berathung.

Bej๘I. = Bejuluß.

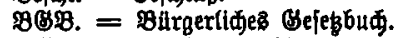

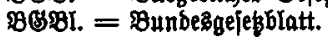

Bitermann $=$ B., Brtbatredt $\mathbf{~}$. Bolfzei in \$reufert, Berlin 1897. BI. f. [0z. Br. $=$ Blätter für fogiale Bracis in 5 salbjahrôhefter.

BP. $=$ Bundestrath.

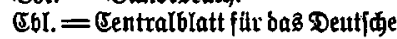
Reid.

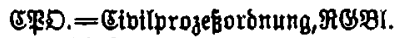
1898 5. 410.

Dep. $=$ Deputation.

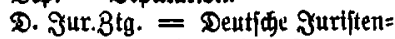
zeitung bon \&aband u. $\mathfrak{x}$.

ES. = EinfittGrungagefe

GIf $=$ Sotfr. $=$ EFlja $\beta=$ =othringen.

eb. $=$ ebentuell.

faabr. = ₹abrit.

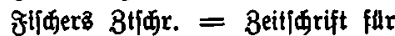
\$rarts u. Bejesgebung ber \$er= waltung, zunähít i. Rönigrei⿻ Sadj]en, bont of.

Bej. = Béeses.

Bew.Ber.Bej. $=$ Betwerbegerifts= geies.

Bew.St.Bef. $=$ ßreup. Semerbe= fteuergeie b. 24. Funt 1891 (B5. 205).

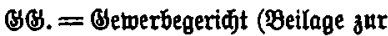
Socialen \$rariş).

BD. = Betwerbeorbnung.

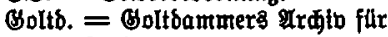
Eirafredit.

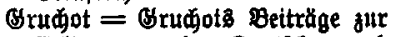

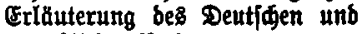

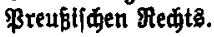

BS. = \$reuß. Befesjanmlung.

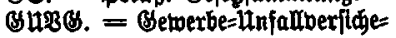


rungêge|ek b. 30. Şunt 1900 ,

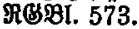

(SPBL $=$.\$efcB= unb Berordnung $8=$ blatt.

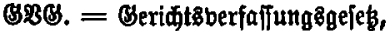

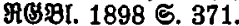

5owl. = \$anbwerfizammer.

Бef]. = Бe[fif

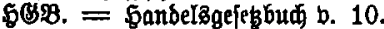

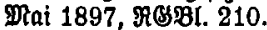

SMBZI. = Minifterial=mlatt ber preus. Gandelä= u. Setwerbeber= waltung.

EMRin. = Eandelosminif́ter.

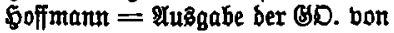
ঔे. छoffunann.

- Drganif. = Die Drganijation beş Gandwertz $2 c$. bon bemjelben, 1897.

\$3. = \$andelobertrag.

Э. = \$nnung.

t. $\mathfrak{u} .=$ im $\mathfrak{u m b e r z i e b e n . ~}$

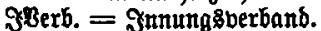

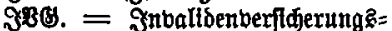

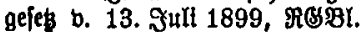
463.

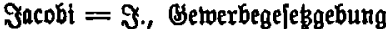
1874.

JDEPI. = Suftizminiftertal6latt.

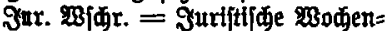
jínift.

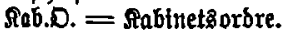

คr. $=$ \$reū. Sommuttalab= gabengejes b. 14. Juli 1893, \&5. 152.

$\mathfrak{H} 8 .=$ Sommtffiortaberidgt.

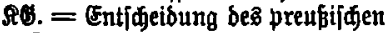
stammergerifits.

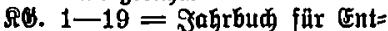

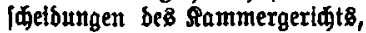
bon Joholn $2 C$.

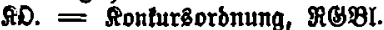
1898 S. 612.

Ramp(s\$. $=$ Sompetenggeridjtô= Gofientiøeibung.

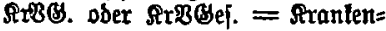

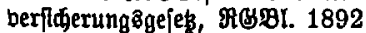
ธ. 417.
Randmann $=\Re$. bon Ranomanns Sommentar zur Setverbeoronung 2. u. 3. פuflage.

\&S. = Sandgeridit.

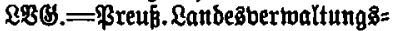
gejes v. 30. Jult 1883, ss. 195.

Pranory $(\mathbb{D}:=$ Seib) $=$ Der cibil=

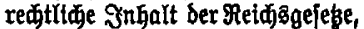
3. भuff., 1898.

Mat. = Materialien von Gejeben.

DEPI. = Minifterialblatt, inşbef. preubifigeas fär ble innere Ber= waltung.

DRE. = DAtnifterialerlaß.

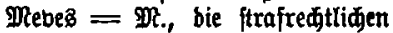
Beftintmungen ber Deutiden (Se= werbeorbnung, in Bezolos Bejes=

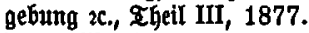

Piit. = Minifter.

Reutanp = Augabe \$. GD. bon $\Re$.

Rov. $=$ Rovelle.

Db.\&abr. Ranbešger. = Entjhet

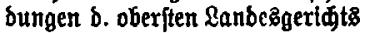
fitr Bayert.

$-1 \mathrm{ff} .=$ beren offizielle samm= lung.

D̈ff. ober Bffentl. = sffentliø.

D\&\$. = Entideibung beŝ oberiten (Serifitahofô (Babern).

DRC. = Entføeioung besి Dber= lanbesigeriøta.

DRS. Minchen $1-8=$ offiztelle Gammlung bon Entidjeibungen beకి DRG. Ditundien.

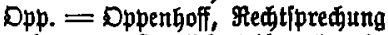
bes preuti. Dbertrifumalo in Strafiadien, 1-20.

DIr. = Entiøjeibungen beş fruberen preus. Dbertribunala.

$-1 \mathrm{fl} .=$ beren offitielle Samm= lung.

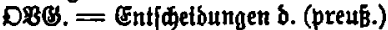
Dherberwaltungasgertats.

-1 ff. = beren offtielle Samm= Iung.

\$ol. = ßolizel, polizeilid.

\$ol.\$răf. = \$olizeipräjtbent.

\$r. ober preup. $=$ preußifa. 


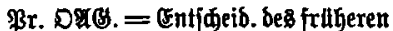

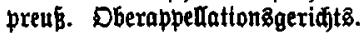
\$rot. $=$ Brotololfe.

\$BRI. = \$reuß. Berbaltungâblatt.

Reg.:BI. = Regierungąblatt.

Reger = Entififetbuttgen ber Beridite u. Bertwaltuntgasbegörber $2 c$. bon A. Meger, $98.1-18$ u. Er= günzungổbanb.

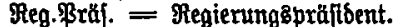

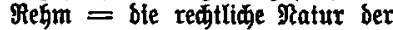
(Betwerbatonzefion bon $\Re ., 1889$.

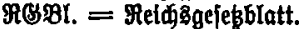

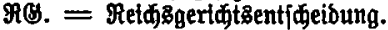
- es. = Dffizielle Sammllung $j \mathrm{ol}=$

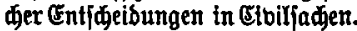

- Stf. = beg̈gl. in Straffadent.

- Mipr. = Rectipredurng bez

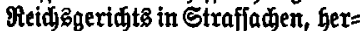
auşgegeben bon ben Mittgliebernt

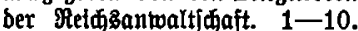

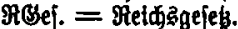

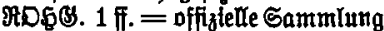
bon Entideibungen bes fritheren

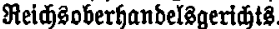

$\Re \mathfrak{T} .=\Re$ etwğtag.

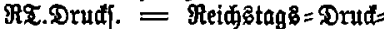
jadien.

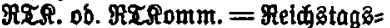
tommifflor.

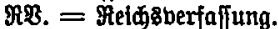

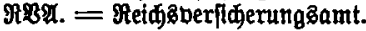

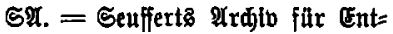
fajeibungen ber oberiten Beridtz= Gôfe Deutifiandas.

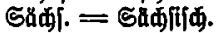

Edentel = Somtmentar zur be werheorbnung bon פor. Sdienter, 2. Iuflage, 1894.

Sditder = beğgl. bon b. Sditder, 3. u. 4. Iuffage, 1892 u. 1898.

Sebbel = Gemerbe=\$olizeirefit $x$. yon Dr. MRar Sebdel, 1881, Sc=

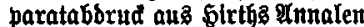
1881.

Soz. \$r. = Soztale \$rartis (Beit= (1)rift).

Sfto. = Die Selbftbertwaltung (Beit= [d fifift).

Sten. Ber. $=$ Stenograpbilije Be= rtabte.

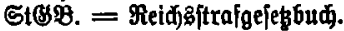

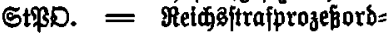
nung.

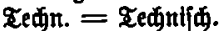

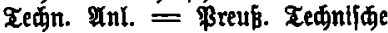
थnTeiturg.

$\mathfrak{u} .=\mathfrak{u}$ rtheil.

$\mathfrak{B} .=$ Berorbnung.

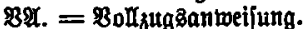

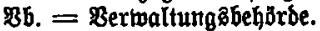

Bertr. $=$ Bertrag.

Berw.Ber.Bef. $=$ Berwaltungg=

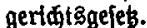

B(5). = Entf heib. eineb Bertwal=

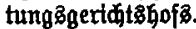

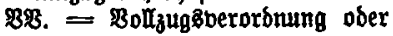
=berfilgung.

B3Gej. = Bereingి olgeję b. 1.

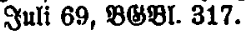

$235 .=$ modjenblatt.

$23 \mathrm{gid} .=23$ anbergewerbejdetr.

$234 t$ trtb. $=$ W3itttembergi

BG. = ßreuß. 8uftänbigleitzoesch vom 1. भuguft 1883, GS. 237.

Die Rommentare bon b. Ranbmann, Sdjentel, b. Sdjider $2 c$. find

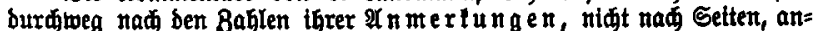
gefubst. 